\title{
MIRTA YÁÑEZ: A LITERATURA DE AUTORIA FEMININA EM CUBA
}

\author{
Maria de Fátima Moreira Peres
}

\begin{abstract}
RESUMO: A entrevista abaixo é fruto de um encontro com a escritora cubana Mirta Yáñez, quando esteve no Brasil em 2010. Com 36 publicações, já foi premiada diversas vezes, inclusive com o prêmio Förderpreis der Iniciative LiBeraturpreis, em Frankfurt, Alemanha, no ano de 2001. Quem vê aquela mulher pequena, frazina, mas extremamente bem articulada e muito atenciosa com todos, não imagina a sua grandeza e contribuição para a literatura cubana e mundial. Ela conta, nessa entrevista, um pouco da sua produção literária.
\end{abstract}

PALAVRAS-CHAVES: Literatura. Cuba. Mulheres. Poesia. Hispânicas.

\section{MIRTA YÁÑEZ: LA LITERATURA DE AUTORÍA DE LA MUJER EN CUBA}

RESUMEN: La siguiente entrevista es el resultado de un encuentro con la escritora cubana Mirta Yáñez, cuando en una visita a Brasil en 2010. Con 36 publicaciones, ha sido galardonada en varias ocasiones, incluyendo el premio Förderpreis der LiBeraturpreis en Frankfurt, Alemania en 2001. Uno que ve esa mujer pequeña, frágil, pero muy bien articulada y muy amable, no se da cuenta de su grandeza y de su contribución a la literatura cubana y universal. Ella dice en esta entrevista un poco de su producción literaria.

PALABRAS CLAVE: Literatura. Cuba. Mujeres. Poesía. Hispánicas.

\section{MIRTA YANEZ: LITERATURE AUTHORSHIP OF WOMEN IN CUBA}

ABSTRACT: The interview below is the result of a meeting with Cuban writer Mirta Yañez, when on a visit to Brazil in 2010. With 36 publications, she has been awarded several times, including the award Förderpreis der LiBeraturpreis Initiative in Frankfurt, Germany in 2001. Who sees that little and frail woman but extremely well articulated and very helpful does not realize her greatness and contribution to Cuban and world literature. She says in this interview, a little about her literary production.

KEYWORDS: Literature. Cuba. Women. Poetry. Hispanic.

Mirta Gloria Yáñez Quiñoá, mais conhecida no mundo literário como Mirta Yáñez, nasceu no dia 4 de abril de 1947, em Havana, Cuba. É escritora, jornalista e professora. Ela é uma mulher pequena, bem franzina, simpática e expressiva. Mas quem a conhece sabe que por trás daqueles poucos centímetros de ser humano existe uma mulher gigante, batalhadora. O currículo de Mirta é extenso, mais de 20 páginas, que representam os anos de dedicação e amor à literatura, às letras, à academia cubana. É formada em Inglês, Russo e Italiano, além de ter 
estudado e conhecer bem o idioma Francês. A escritora cubana tem diversos livros publicados. E entre os temas, por ela abordados, a mulher tem um destaque especial, principalmente sobre o discurso feminino e a poesia de autoria feminina. Segundo Mirta Yáñez, em Cuba, as mulheres seguem livres para serem escritoras, mas ainda é um campo em que os homens continuam gozando de privilégios. Ainda prevalece o machismo sobre a obra literária produzida em Cuba. Mas sempre que pode, em suas andanças pelo mundo afora, fazendo palestras, dando aulas, ou mesmo lançando livros, Mirta destaca a força e o talento das mulheres cubanas. É a sua forma de contribuição para o reconhecimento da produção literária de autoria feminina de seu país. Porém, mesmo com essa desigualdade entre as produções literárias, no que se refere a oportunidades econômicas e políticas, Cuba, segundo uma pesquisa realizada pelo World Economic Forum (WEF) é considerado o $20^{\circ}$ país do mundo com menor índice de desigualdade de gênero. Entre os países da América Latina e Caribe, Cuba supera todos eles. Ou seja, no coletivo, no jurídico, as mulheres se aproximam dos homens em igualdade, mas no particular, ainda têm alguns passos para evoluir, ainda que seja inegável a participação das mulheres na revolução cubana e o respeito que existe pela figura feminina naquele país. A entrevista que se segue foi feita após a sua participação em um seminário sobre literatura de autoria feminina na cidade de Divinópolis, no Centro-Oeste de Minas Gerais, Brasil, em 2010. Além de dar seu depoimento sobre o fazer literário feminino em Cuba ela lançou ainda o livro do irmão Alberto Yáñez que havia falecido há pouco tempo. O livro - Trocando Gato por lebre ou menino por vaca - dedicado ao público infanto-juvenil tem tradução de Bartolomeu Campos de Queirós e é uma obra de arte, não só da escrita, do conteúdo, mas também como um produto pensado com toda a qualidade editorial e dedicação daqueles que amam a arte de escrever.

Gloria Mirta Yáñez Quinoá, mejor conocida en el mundo literario como Mirta Yáñez, nació el 4 de abril de 1947, en La Habana, Cuba. Ella es una escritora, periodista y maestra. Ella es una mujer menuda y joven, simpática y expresiva. Pero, quién la conoce sabe que detrás de esos pocos centímetros de ser humano hay una mujer gigante, una luchadora. El curriculum vitae de Mirta es demasiado largo, más de 20 páginas, que representa a los años de dedicación y amor por la literatura, por las letras, por la Academia Cubana. Se graduó en Inglés, Italiano y Ruso, y se ha estudiado y conoce bien lo idioma Francés. Esa escritora cubana ha publicado varios libros. Y entre los temas abordados por ella, la mujer se pone de relieve, sobre todo en el discurso femenino y la poesía escrita por mujeres. De acuerdo con 
Mirta Yáñez, en Cuba, las mujeres son libres para convertirse en escritoras, pero la literatura en general sigue siendo un campo en el que los hombres siguen disfrutando de los privilegios. El sexismo sigue existiendo sobre la obra literaria producida en Cuba. Pero cada vez que puede, en sus viajes por el mundo dando conferencias, enseñando, o incluso en la publicación de libros, Mirta destaca la fuerza y el talento de las mujeres cubanas. Es su forma de contribución al reconocimiento de la producción literaria de la autoría femenina de su país. Pero a pesar de esta desigualdad entre las producciones literarias, con lo que se refiere a las oportunidades económicas y políticas, Cuba, según una encuesta realizada por el Foro Económico Mundial (FEM) es considerado el $20^{\circ}$ país del mundo con la menor desigualdad de género. Entre los países de América Latina y el Caribe, Cuba supera a todos ellos. Es decir, colectivamente, en la ley, las mujeres acercarse a los hombres en igualdad de condiciones, pero en particular, todavía tiene algunos pasos para evolucionar; sin embargo, es innegable que la participación de las mujeres en la revolución cubana y el respeto que existe para la figura femenina en ese país. La siguiente entrevista fue realizada después de su participación en un seminario sobre la literatura de autoría femenina en la ciudad de Divinópolis, en el Centro-Oeste de Minas Gerais, Brasil, en 2010. Además de dar su declaración sobre la producción literaria femenina en Cuba, Mirtá presentó también un libro de autoría de su hermano Alberto Yáñez que había muerto recientemente. El libro - Trocando Gato por lebre ou menino por vaca - dedicado a los niños y los jóvenes tiene traducione de Bartolomeu Campos de Queirós y es una obra de arte, no sólo de la escritura, en su contenido, sino también como un producto diseñado con toda la calidad editorial y la dedicación de aquellos que aman el arte de escribir.

Gloria Mirta Yáñez Quinoá, better known in the literary world as Mirta Yáñez, was born on April 4, 1947, in Havana, Cuba. She is a writer, journalist and teacher. She is a little and frial woman, friendly and expressive. But who knows her knows that behind those few feet of human being there is a giant woman, a fighter. Mirta's resume is extensive, more than 20 pages, representing the years of dedication and love to the literature, letters and Cuban Academy. She graduated in English, Italian and Russian, and have studied and knows well the French language. This Cuban writer has published several books. And among the topics addressed by her, the woman is in special focus, especially on the female discourse and poetry authored by women. According to Mirta Yáñez, in Cuba, the women are free to become 
writers but literature is still a field in which men continue enjoying privileges. Sexism still prevails about the literary work produced in Cuba. But whenever she can, in her travels around the world, lecturing, teaching, or even publishing books, Mirta highlights the strength and talent of Cuban women. It is her way of contribution to the recognition of the literary production of female authorship of her country. But even with this inequality between the literary productions, with regard to economic and political opportunities, Cuba, according to a survey by the World Economic Forum (WEF) is considered the 20th country in the world with the lowest gender inequality. Among the countries of Latin America and the Caribbean, Cuba surpasses them all. That is, collectively, in law, women approach men on equal terms, but in particular, still have some steps to evolve, yet it is undeniable the participation of the women in the Cuban revolution and the respect that exists for the female figure in that country. The following interview was done after her participation in a seminar on the literature of female authorship in the city of Divinópolis, in the Central West of Minas Gerais, Brazil, in 2010. Besides giving her statement about the female literary production in Cuba she has also introduced a book written by her brother Alberto Yáñez who had recently died. The book - Trocando Gato por lebre ou menino por vaca - dedicated to children is translation of Bartolomeu Campos de Queiros and is youth is a work of art, not only of writing and in its content, but also as a product designed with all the editorial quality and dedication of those who love the art of writing.

\section{¿Quién es Mirta?}

Mirta: Mi nombre es Mirta Gloria Yáñez Quiñoá. Soy, ante todo, una escritora. Cubana, descendientes de gallegos y asturianos, amante de perros y gatos, del signo de Aries, aspiro a la honestidad ante todos los hechos de la vida. Soy Doctora en Ciencias Filológicas pela Universidad de La Habana, Cuba em 1992. Licenciada en Lengua y Literaturas Hispánicas de la Escuela de Letras y Arte de la Universidad de La Habana em 1970 e profesora Auxiliar de la Universidad de La Habana, Cuba em 1985. Me gradué en estudios titulados de Inglés, Ruso e Italiano. También ha estudiado y conoce el Francés. Soy miembro de las organizaciones: Unión de Periodistas de Cuba (UPEC) desde 1987 e Unión de Escritores y Artistas de Cuba (UNEAC) desde 1977.

¿Cuáles son los principales temas de investigación académica? 
Mirta: Mi principales temas de investigación académica: narradoras cubanas y el discurso femenino en Cuba, poesía cubana escrita por mujeres, desarrollo histórico y estético del cuento: el proceso de la narración breve en América Latina y Cuba, romanticismo en hispanoamérica, poesía romántica del siglo XIX hispanoamericano e literatura prehispánica.

\section{¿En cuáles Universidades donde ha colaborado con cursos y conferencias?}

Mirta: Universidad de La Habana, (Cuba). Universidad de los Andes, Mérida, (Venezuela). Universidad Autónoma de Puebla, El Colegio de México, Universidad Autónoma de México, Universidad Iberoamericana, (México). Universidad Autónoma de Nicaragua, (Nicaragua). Universidad de Panamá, (Panamá). Universidad Estadual de Río de Janeiro, (Brasil). Universidad de Poitiers, Universidad de Burdeos, Universidad de Nantes, Universidad de la Sorbona, París VIII, (Francia). Universidad de Sassari, Universitá Ca`Foscari, Universitá degli Studi di Milano (Italia). Universidad de Manchester, Universidad de St. Andrews, (Inglaterra). Universidad de Kassel, Universidad de Gotinga, Universidad de Hannover, Universidad de Bremen, (Alemania). Universidad Nacional de Tucumán, Universidad Nacional de Salta, (Argentina). Vassar College, Marist College, Florida International University, University of Massachusetts, York College, North Georgia College, Appalachian University, Wofford College, St. Thomas University, Stanford University, UNCA, Boone University, Syracuse University (USA). Universidad de Puerto Rico, Recinto de Cayey, Recinto de Río Piedras, Recinto de Bayamón, (Puerto Rico).University of Toronto, Middlesex College, University of Guelph, York University, Ryerson Politechnic University (Canadá), Universidad de Graz, Austria, Fundaçao Educacional de Divinopolis y en la Catedra Unesco de Leitura PUCRio, entre otras.

\section{¿Cuántos libros ya publicado e cuáles?}

Mirta: Tengo 36 obras publicadas. Sangra por la herida (novela), Ediciones UNION y Ed. Letras Cubanas, La Habana, 2010. Havana is a really big city and other short stories (cuentos), Ed. Cubanabooks, USA, 2010. El búfalo ciego y otros cuentos (compilación de cuentos), Ediciones UNIÓN, La Habana, 2008. Faux Papiers Falsos documentos (cuentos, edición bilingüe), Ed. MEET, Saint-Nazaire, Francia, 2007. Serafín y las aventuras en el Reino de los Comejenes (cuento infantil), Colección Dienteleche, Ediciones UNIÓN, La Habana, 2007. La fiesta de los caballitos (novela infantil, edición mexicana de Serafín y sus 
aventuras con los caballitos), Ed. Progreso, México D. F., 2006. Cenicienta (versión teatral), Ed. Gente Nueva, La Habana, 2006. Del azafrán al lirio, (recopilación de textos variados), Ed. Extramuros, La Habana, 2006. El Matadero: un modelo para desarmar (ensayo), Ed. Letras Cubanas, La Habana, 2005. Falsos documentos,(cuentos), Colección Vagabundo del Alba, Ediciones UNIÓN, La Habana, 2005. Havanna ist eine ziemlich grosse stadt (cuento), Ed. Atlantik, Bremen, Alemania, 2001. Camila y Camila, (testimonio), Ediciones La memoria, Centro Cultural "Pablo de la Torriente Brau", La Habana, 2003. Un solo bosque negro, (compilación poética) Ed. Letras Cubanas, La Habana, 2003. Serafín y sus aventuras con los caballitos (novela infantil), Ed. Gente Nueva, La Habana, 2003 (tercera edición). Cubanas a capítulo (ensayo), Ed. Oriente, Santiago de Cuba, 2000. El diablo son las cosas, (cuento, segunda edición) Ed. Letras Cubanas, La Habana, 2000. Narraciones desordenadas e incompletas, (narraciones) Ed. Letras Cubanas, La Habana, 1997. Algún lugar en ruinas (poesía), Ediciones UNIÓN, La Habana, 1997. Poesía casi completa de Jiribilla el conejo (poesía para niños), Ed. Gente Nueva, La Habana, 1994. Todos los negros tomamos café y otros cuentos (cuento), Edición del autor, Tucumán, Argentina, 1993. El diablo son las cosas (cuento), Edición del autor Tucumán, Argentina, 1993. Una memoria de elefante (testimonio), Ed. Abril, La Habana, 1991. La narrativa romántica en Latinoamérica (ensayo), Colección “Giraldilla”, Ed. Letras Cubanas, La Habana, 1990. Notas de clase (poesía), Colección "La barca de papel”, Imprenta de la Dirección de Información, Ministerio de Cultura, La Habana, 1989. El diablo son las cosas (cuento), Ed. Letras Cubanas, La Habana, 1988. Serafín y sus aventuras con los caballitos (novela infantil, segunda edición, ilustraciones de la autora), Ed. Gente Nueva, La Habana, 1987. Poemas (poesía), Ed. Embalaje, Colombia, 1987. El mundo literario prehispánico (ensayo, en colaboración), Imprenta Universitaria, La Habana, 1986. Las visitas y otros poemas (poesía), Ed. Letras Cubanas, La Habana, 1986. La hora de los mameyes (novela), Ed. Letras Cubanas, La Habana, 1983. "Yo soy Jack Johnson" (relato), en Cuentos de boxeo, Ed. Arte y Literatura, La Habana, 1982. La Habana es una ciudad bien grande (cuento), Ed. Letras Cubanas, La Habana, 1980. Serafín y sus aventuras con los caballitos (novela infantil), Ed. Gente Nueva, La Habana, 1979. Todos los negros tomamos café (cuento), Ed. Arte y Literatura, Instituto Cubano del Libro, La Habana, 1976. Quiénes eran los aztecas (divulgación docente), Centro de Información Científico-Técnica de la Universidad de La Habana, La Habana, 1974. Las visitas (poesía), Imprenta Universitaria, Universidad de La Habana, La Habana, 1971. 


\section{¿Tienes textos literarios de ficción publicados en publicaciones periódicas el} extranjero e en cuáles?

Mirta: Si. "Niemand ruft aus dem Wald/ Wie Enoch Soames", Literatur Nachrichten Afrika Asien Lateinamerika, Frankfurt, 2010, no. 104. "Pájaro de mal aguero", Soglie americane, “Quale america? Soglie e culture di un continente”. Volume 1, Mazzanti Editori, Venezia, 2007. “Periodización", “Objetos” y “Credulidades”, Meridian 42, Indiana University Press, 2004. "Kid Bururu et les cannibales", MEET, Saint-Nazaire, France, 2003, no. 7. "Nada salvo el aire", Feminaria, Buenos Aires, julio de 2002, año XV, no. 28-29. "Todos los negros tomamos café", Revista de la Universidad, Tegucigalpa, octubre, noviembre, diciembre 2001, año 2, no. 5. "Nada salvo el aire", Cuba: una literatura sin fronteras, Iberoamericana, Madrid, 2001, Vervuert, Frankfurt, 2001. "Dos poemas", Teluria, Cochabamba, octubre 2000, no. 7. "Version Originale" y "L'Ile dans le Bagages", (cuento y artículo), Encuentros, France-Amérique Latine, Bordeaux, 2000. “Anagnórisis”, Donaire, Inglaterra, noviembre 2000, no. 15. "Memorias desde La Habana”, Txalaparta, 8 zenbakia, País Vasco, 2000. "Versión original”, “Barcarola, Revista de creación literaria, Albacete, junio 1997, no. 53. "Der blinde Büffel”, (cuento), Tranvia, Revue der Iberischen Halbinsel, Heft 43, Alemania, Dez.'96, "Versión original”, (cuento), Xicóatl, Austria noviembre-diciembre 1996, 5 año, no.28. "El diablo son las cosas", (cuento), Crítica, Revista cultural de la Universidad Autónoma de Puebla, México, Nueva época, juniojulio 1996, no. 62. "El diablo son las cosas", (cuento), Tierra adentro, Publicación del Consejo Nacional para la Cultura y las Bellas Artes y el Instituto Nacional de Bellas Artes, México, marzo-abril 1993, no. 64. "Chronology", (poema), Women Poets of the Caribbean, The Literary Review, Fairleigh Dickinson University, USA, summer 1992, volume 35, number 4. "Poema", Napjaink, Hungría, 9 szeptember, año XVIII. "El búfalo ciego", (cuento), Casa del Tiempo, México, abril 1989, vol. VIII, no. 84. "Conferencia", (poema), La palabra y el hombre, Universidad Veracruzana, México, abril-junio 1988, no. 66. "Il bufalo cieco”, Noi Donne, Italia, gennaio 1988, anno 43, no. 1. "Cinco de la mañana", (poema), Zurda, México, primer semestre de 1988, no. 3. "Wymiana winna byc szersza", (poema y entrevista), Polska Micoer, Polonia 22I-1987. "Generational task", "Context of Sor Juana", (poemas), “Conditions: fourteen”, USA, 1987. "Poeci swiata", (poema), Polska micoer, Polonia, 1986, no. 42. "Jutío", (fragmento de La hora de los mameyes), Renie Svetovej Literatury, Checoslovaquia, 1986, no. 2. "First chronicle", 
(poema), Social Test, USA, fall 1986. "La Habana es una ciudad bien grande”, (cuento), Puesto de Combate, Bogotá, marzo 1976, año III, no. 9.

\section{¿Usted tiene otros trabajos publicados?}

Mirta: Sí, diversos artículos, críticas y trabajos periodísticos publicados en Cuba e prólogos en los textos: Aurelia Castillo: ética y feminismo, Mercedes Valdés Estrella, Publicaciones Acuario, Centro Félix Varela, La Habana, 2008. De silencios y lunas, Rolando López del Amo, Ed. Letras Cubanas, La Habana, 2005. Oficio de difuntos, Arturo Uslar Pietri, Ed. Arte y Literatura, La Habana, 1988. Cuentos de abuelas, Roberto Pérez León, Imprenta Universitaria, La Habana, 1985. El guaraní, José de Alençar, Ed. Casa de las Américas, La Habana, 1983. Por entre los sueños cantos, José A. Gutiérrez, Imprenta Universitaria, La Habana, 1980. Edición cubana de El corazón es un cazador solitario, Carson McCullers, Ed. Arte y Literatura, La Habana, 1976. Edición cubana de Un yanqui de Connecticut en la corte del rey Arturo, Mark Twain, Ed. Arte y Literatura, La Habana, 1976. Colección de poemas, Imprenta Universitaria, La Habana, 1975.

Además de estos los testimonios en: Asturias en la memoria, Miriam Rodríguez Betancourt, Asturias, 2008. Sembró maestros, (Homenaje a Rosario Novoa), Universidad de La Habana, en 1994. Los ensayos en: Con el lente oblicuo, Aproximaciones cubanas a los estudios de género, "El discurso femenino finisecular en Cuba: Aurelia del Castillo y otras voces en torno al 98", Editorial de la Mujer, Instituto de Literatura y Lingüística em 1999. Mujeres latinoamericanas: Historia y cultura Siglos XVI al XIX, ("Cecilia Valdés, la heroína corriente, la heroína solitaria") Serie Coloquios, tomo II, Ed. Casa de las Américas y Universidad Autónoma Metropolitana, Cuba-México en 1997.

\section{¿Algun textos inédito?}

Mirta: Si: "Sobre el Popol Vuh", para la Enciclopedia Juvenil de la Editorial Gente Nueva.

\section{¿Cuando usted se convierte en un escritor?}

Mirta: Creo que nací con el "bichito" de las letras. Mi padre era periodista, y tenía un tío abuelo que escribía poemas. Empecé a escribi a los siete años. También quería ser pintora, pero las letras me halaron más. Ahora bien, la clara conciencia del destino de escribir fue 
aproxidamente a los 14 años, en la adolescencia, decidí que lo único que podía hacer en la vida era escribir.

\section{¿Cuando publicó su primer libro? ¿Cómo se llama? Hable sobre lo que escribió.}

Mirta: Mi primer libro lo publiqué despues de ganar con él un concursi literario de la universidad a los veinte años. Salió unos años despues. Se llama LAS VISITAS, y es un breve cuaderno de poemas. Es el libro que quiero más. Esos poemas los empecé a escribir cuando era estudiante de Letras de la asignatura Historia del Arte, teníamos que recorrer La Habana y ahí empecé a descubrir todos los amores que perdurarían en mi vida: la ciudad, sus gentes, el mar, los animales callejeros, el primero amor...

\section{¿Cómo funciona la inspiración para escribir?}

Mirta: No se si a otros les funciona como a mi... yo tengo que estar trabajando para que se "cuele" la inspiración. Tengo que tener un buen estado de animo, silencio total, ningún agobio de otras tareas pendientes, y trabajando. Entonces entra a funcionar la inspiración.

\section{¿Cuáles son los temas (historias) de sus libros?}

Mirta: Los temas son la vida comun y corriente, en mis cuentos. Y de hecho en la poesía, y en la novela también. En el ensayo tengo dos temas fundamentales: la literatura escrita por mujeres en Cuba y el romanticismo latinoamericano del siglo XIX. En la literatura para niños trato de partir de un hecho fantasioso y que por el camino desarrolle alguna idea noble a compartir con los niños. En mi novela más reciente SANGRA POR LA HERDA trato de contar la confusión de mi generacion en el devenir de la historia, con hechos y asuntos de todos los días que bordean a veces la tragicomedia.

\section{¿Es difícil para una mujer cubana a ser escritor?}

Mirta: Llegar a escribir no, puesto que es un acto de libre elección. Ahora bien, desarrollar su obra en medio de un contexto machista y excluyente, pues, sí, a algunas se nos hace difícil. Pero ya eso paulatinamente ha ido cambiando, aunque todavia los jurados, los directores de revistas, los consejos asesores, y otras acciones dentro del campo literario, independiente del éxito o los progresos de las escritoras, siguen siendo terreno de los hombres. 


\section{¿Hay un tema tabú en Cuba? Ejemplo: sexo, política, etc ...}

Mirta: Eso depende. De temas tabus se ha ido al otro extremo: a supuestamente una libertad sin mucha responsabilidad y exigencia estetica. Estoy pensando en "libros" que caen en la chabacanería o palabras malsonantes por el simple hecho de hacerlo. En cuanto a la politica las autoridades editoriales aspiran a que los libros publicados en Cuba, ya que hay tanta crisis económica y tan poco papel, respondan a la ideología general. De todas maneras, en todas partes del mundo se usa de esta misma criba selectiva. Por ejemplo, a los cubanos que vivimos dentro de Cuba se nos dificulta mucho al acceso a determinadas areas de venta en

\section{¿Una mujer cubana lee mucho? ¿Qué tipo de literatura?}

Mirta: Los cubanos y las cubanas leemos mucho. No sabría decirte que tipo de literatura leen los demás, pero si te cuento que en las Ferias del Libro son verdaderas fiestas a tal punto que yo les digo LA FURIA del libro. Gustan las novelas policiacas, las biografías, en general la narrativa que refleje los problemas vivos de nuestra sociedad. Tambien se vende y se agota rapido la literatura para niños. El libro que siempre hay que reeditar es La edad de oro de José Martí.

\section{¿Cuál es tu estilo?}

Mirta: Mi estilo es directo, con el lenguaje del habla cubana, eso si, trato de mantener un rigor en el uso del lenguaje. Digamos que mi caracteristica estilistica es la ironía, la mezcla del drama con el humor. Y aparentando una sencillez que esconde honduras. Ya el lenguaje poetico ha ido quedando atrás y me quedó con la prosa más clara que pueda alcanzar.

\section{¿Se podía leer un texto suyo?}

Mirta: Sí, voy a leer: Nadie llama de la selva.

El perro había quedado atrás. Quizás no se llamaba Buck, aunque tampoco leía periódicos, así que no sospechó nada. La casa fue cerrada y el jardín se detuvo tras una cerca de dos metros de altura, cubierta a tramos por una enredadera. El perro estaba de pie en el portal, vigilante, con las orejas enhiestas y en actitud de espera. Desde la calle no se le 
podía distinguir mucho. Desde la ventanilla del ómnibus se veía no sólo al perro, sino el sello oficial que clausuraba la casa.

El perro era blanco, con algunos mechones oscuros en el pecho y en el lomo, de pelo corto y lustroso, bien cuidado. En los primeros días se afirmaba en las cuatro patas con seguridad y altivez. No olfateaba el viento ni se movía, simplemente esperaba. La casa era una de esas añosas de El Vedado, ya despintada y con aires de decadencia. Sin embargo, el jardín se notaba verdecido y daba muestras de haber sido podado en fechas recientes. El soplo de abandono que se iría posesionando de todos sus recovecos, todavía no había borrado la memoria de las manos que una vez lo atendieron.

Al cabo de unos días, el perro continuaba en igual posición, al lado de la puerta principal. Sin duda no quería moverse para ser el primero en notar el regreso de quienes él sabía que tenían derecho a entrar en la casa y reanudar la vida, la única vida que el perro había conocido. Se mantenía en su sitio, con la misma expresión orgullosa, confiada, aunque su bella estampa comenzaba a deteriorarse. Podría pensarse que estuviera ya impaciente, había dejado de gustarle el juego, como broma ya bastaba.

Una semana más tarde, el perro acusaba algún desconcierto. ¿Qué pasaba? ¿Qué podía haber hecho mal? ¿Por qué sus amos, sus dioses, no regresaban? Seguía de pie y mirando fijamente hacia el punto exacto por donde había visto a su familia por última vez, pero ya con cierta inquietud y fatiga, con toda certeza también hambre y sed. No le importaba mucho, en realidad, la falta de alimento. Ni tan siquiera no poder entrar a su cubil predilecto, hacerse un ovillo, suspirar y dormirse con el corazón en calma. Toda su pequeña cabeza estaba concentrada en entender a qué se debía aquel castigo que no creía merecer.

El perro no había oído hablar de Buck, así que no se sentía un héroe. No había visto nunca nieves, ni trineos, ni ventisqueros, ni aquellas eran las heladas comarcas del Klondike. Nadie le había pegado nunca con un palo. Cuando paseaba por el barrio lo llevaban con unas cómodas correas que más bien lo hacían sentirse protegido y ni siquiera tenía idea de que otros perros como él podían matarse a mordidas. Esta era la casa donde había vivido siempre desde que lo trajeron como cachorro. Detrás de la puerta sellada quedaron sus escondrijos, su pozuelo de agua y el cacharro de comer. Aunque todo eso era lo de menos. ¿Por qué lo habían abandonado?

Quince días después permanecía aún de pie, con resignación, como víctima de un error incomprensible. Pero el agotamiento terminó por acorralarlo y se vio obligado, a pesar suyo, 
a reclinarse contra la puerta. Se le cerraron los ojos y soñó. Soñaba que la familia regresaba, la casa se llenaba de voces y ruidos conocidos, las ventanas se abrían al sol de la mañana y se despertó gozoso, dando un ladrido que se transformó en silencio y en jalones de ira. Se sintió engañado, furioso, de nuevo estaba allí la pesadilla de la casa cerrada, del jardín que se secaba como su propio cuerpo. Ya no se preguntaba qué había hecho mal, sólo quería que el castigo terminara.

Pasado un tiempo, tenía un aspecto miserable, aunque se mantenía todavía mirando hacia al mismo lugar. Las orejas alertas eran el único residuo que quedaba de su prestancia de los primeros días. Tenía el cuerpo enjuto y consumido, el pelo viscoso y la mirada vidriosa. La espera estaba llegando a su fin y algo parecido a la piedad, al perdón, entraba en su leal corazón de perro. Ellos, sus dioses, sabrían por qué lo habían hecho.

Hortensia, la mamá de Julia, vivía en el último piso del edificio vecino a la casa del perro. La escalera no tenía bombillos y Hortensia había ido perdiendo la vista, así que no salía nunca y sólo se sentaba en el balcón a escuchar los sonidos de la calle. Hortensia, como Buck, tampoco leía periódicos. Le hubiera gustado escuchar el radio, sus novelones, como decía Julia, pero estaba roto hacía mil años. Antes de que se muriera, Manchita era su compañía. Hortensia le daba los buenos días, la regañaba y, a veces, le conversaba sus problemas. Con Manchita la existencia transcurría más entretenida. Hortensia la extrañaba tanto, qué se le iba a hacer, si ya no podía ni con ella misma, dime tú, cómo cuidar de otro perrito. La vecina que la ayudaba de vez en cuando nunca hablaba mucho, tenía sus propias tribulaciones, y gracias que venía a airear la casa y a traerle los mandados de la bodega. A Hortensia le daba hasta vergüenza molestarla y pedirle que, por favor, le leyera las cartas de la hija que, de tanto en tanto, llegaban de la Argentina. Cuando Julia le mandaba uno de aquellos paqueticos con jabones y la medicina para el corazón, Hortensia le regalaba los jabones a la vecina.

Le hubiera gustado también escuchar la voz de Julia, pero, ave maría santísima, mira que las llamadas de ese lugar tan lejano eran caras. Y pasaban los años, y seguían pasando los años, en espera de que vinieran tiempos mejores. Bendito sea el cielo que la medicina y los jabones nunca le faltaban. Y, por suerte, estaba casi ciega, así que no podía distinguir al perro.

Un mes más tarde el perro ya no estaba. No lo habían vencido las nevadas, ni los lobos, ni el hambre, sino aquella tristeza que le impedía hacer otra cosa que seguir cuidando la 
casa y esperar, solitario, el regreso. (Mirta Yáñez, Falsos documentos, La Habanna: editorial UNION,,2007, pags 45 a 47)

\section{REFERÊNCIAS:}

Entrevista realizada com a escritora em 2010, na Fundação Educacional de Divinópolis (MG). Entrevista con la escritora en 2010, la Fundación para la Educación de Divinópolis (MG).

VASSI Cássia. A mulher cubana e sua sociedade: da independência à revolução. São Paulo: Universidade Estadual Paulista, 2007.

QUIÑOÁ, Mirta Gloria Yáñez. Falsos documentos, p. 45 a 47. La Habanna: Editorial UNION, 2007.

RECEBIDO EM: 07 de maio de 2012 APROVADO EM: 15 de junho de 2012 